\title{
A MORSE LEMMA FOR DEGENERATE CRITICAL POINTS WITH LOW DIFFERENTIABILITY
}

\author{
ADRIANO A. DE MOURA AND FAUSTO M. DE SOUZA
}

Received 30 June 2000

We prove a Morse type lemma for, possibly degenerate, critical points of a $C^{1}$ function twice strongly differentiable at those points, which allows us to recover, for Finsler metrics, the theorem of Gromoll and Meyer on the existence of infinitely many closed geodesics.

\section{Introduction}

Morse theory has been successfully applied for proving existence and multiplicity results for extremals of various variational problems. In particular the following beautiful theorem of Gromoll and Meyer (see [3]).

THEOREM 1.1. If $M$ is a compact, simply connected Riemannian manifold whose cohomology is not isomorphic to the one of a compact symmetric space of rank one, that is, a sphere or a projective space, then $M$ has infinitely many closed geodesics (nontrivial and geometrically distinct).

It is still an open problem if the conditions on the cohomology may be withdrawn. On the other side, there are examples of Finsler metrics on rank one symmetric spaces with only a finite number of closed geodesics. One of the differences between the critical points theory for the Finsler and the Riemannian cases is that the Finsler energy is not $C^{2}$. In fact it is twice differentiable at the critical points, but not in general outside the regular curves. Therefore, in order to have a Morse theory for the Finsler case, we need a Morse lemma for functions with such a level of regularity. This was done in [4] for the case of nondegenerate critical points and, as a consequence, they obtained the result of Gromoll and Meyer for the Finsler case with the additional hypothesis that the closed geodesics are nondegenerate circles of critical points in the space of closed curves (nondegenerate in the sense of Bott [1]). 
114 A Morse lemma for degenerate critical points ...

The aim of this paper is to prove a degenerate-critical-point version of the Morse lemma as in [2] with conditions of low differentiability that, although stronger than those in [4], are verified by the Finsler energy. More precisely, let $f: U \subset \mathbb{H} \rightarrow \mathbb{R}$ be a $C^{1}$ function defined on an open set of a Hilbert space $\mathbb{H}$. Suppose that $f$ is twice differentiable at 0 and let $\mathbb{N}$ be the kernel of the symmetric operator $A: \mathbb{H} \rightarrow \mathbb{H}$ given by

$$
\langle A v, u\rangle=\frac{1}{2} d^{2} f_{0}(v, u) \text {. }
$$

If the image $\operatorname{Im}(A)$ is closed, since $A$ is symmetric, $\mathbb{N}^{\perp}=\operatorname{Im}(A)$ and $\mathbb{H}$ is decomposable in $\mathbb{H}=\mathbb{N}^{\perp} \oplus \mathbb{N}$. Thus we can look at $z \in \mathbb{H}$ as $x+y \in \mathbb{N}^{\perp} \oplus \mathbb{N}$. We will prove the following theorem.

THEOREM 1.2. If $f^{\prime}$ is strongly differentiable at the origin, there is a neighborhood $V$ of 0 in $\mathbb{H}$ and a homeomorphism $\varphi: V \rightarrow \varphi(V) \subset \mathbb{H}$ such that

$$
f(\varphi(x, y))=\frac{1}{2}\langle A x, x\rangle+f(g(y), y), \quad d \varphi_{0}=I,
$$

where $g$ is a function $g: V \cap \mathbb{N} \rightarrow \mathbb{N}^{\perp}$.

\section{Proof of Theorem 1.2}

Recall that a function between two Banach spaces, $f: \mathbb{E} \rightarrow \mathbb{F}$, is said to be strongly differentiable at $x$ if $f$ is differentiable at $x$ and

$$
\lim _{y, z \rightarrow x} \frac{r(y)-r(z)}{\|y-z\|}=0,
$$

where $r(y)$ is the rest function of the Taylor's formula for $f$ around $x$. In other words, $f$ is strongly differentiable at $x$ if and only if it is differentiable and, given $\varepsilon>0$, there is a neighborhood of $x$ where $r(y)$ is $\varepsilon$-Lipschitzian (hence so is $f$ ). It is clear also that if $f$ is differentiable on a neighborhood of $x$ and its differential $f^{\prime}$ is continuous at $x$, then $f$ is strongly differentiable at $x$. Moreover, if $f$ is continuous in a neighborhood of $x$ and strongly differentiable at $x$, with invertible differential, then $f$ is invertible around $x$ and the proof is the same as the classical inverse function theorem. Also the corresponding version of the implicit function theorem gives the following proposition.

Proposition 2.1. Using the conditions and notation above, let 0 be a critical point of $f$ and suppose that $f^{\prime}$ is strongly differentiable at the origin. Then there is a continuous function $g: U \subset \mathbb{N} \rightarrow \mathbb{N}^{\perp}$ on an open set $U$ containing 0 such that $(\partial f / \partial x)(g(y), y) \equiv$ 0 and $g(0)=0$. Moreover, $g$ is strongly differentiable at the origin and $d g_{0}=0$.

Remark 2.2. If we write $f(z)=f(x, y)$ and look at the restrictions of $f$ on the planes $\mathbb{N}^{\perp} \times\{y\}$, the above function $g$ gives us a parametrization of the critical points of such restrictions on a neighborhood of the origin in $\mathbb{H}$.

We go now into the proof of Theorem 1.2. Define $h_{1}: \mathbb{N}^{\perp} \oplus U \rightarrow \mathbb{N}^{\perp} \oplus \mathbb{N}$ by

$$
h_{1}(x, y)=x+g(y)+y .
$$


It is clear that $h_{1}$ is strongly differentiable at 0 and that $d h_{1}(0,0)=I$, hence $h_{1}$ is a homeomorphism of a neighborhood $V_{1}$ of the origin of $\mathbb{H}$ onto another neighborhood of 0 , say $W_{1}=h_{1}\left(V_{1}\right)$.

Remark 2.3. Observe that what $h_{1}$ is doing is to move an open set of $\{0\} \times \mathbb{N}$ onto the parametrized surface of "critical points" (in the sense of Remark 2.2) $\{(g(y), y) ; y \in$ $U \subset \mathbb{N}\}$.

Since $\left.A\right|_{\mathbb{N}^{\perp}}$ is an isomorphism, we can write $\mathbb{N}^{\perp}=\mathbb{H}_{-} \oplus \mathbb{H}_{+}$where $\mathbb{H}_{-}$and $\mathbb{H}_{+}$ are the $A$-invariant subspaces where $A$ is negatively defined and positively defined, respectively. This way we have $\mathbb{H}=\mathbb{H}_{-} \oplus \mathbb{H}_{+} \oplus \mathbb{N}$ and $z=x+y$ with $x=x_{-}+x_{+}$. It is convenient to introduce an inner product on $\mathbb{N}^{\perp},(\cdot, \cdot)$, equivalent to the former $\langle\cdot, \cdot\rangle$, which makes the decomposition $\mathbb{H}_{-} \oplus \mathbb{H}_{+}$orthogonal. It suffices to take

$$
\left(u_{-}+u_{+}, v_{-}+v_{+}\right)=\left\langle A u_{+}, v_{+}\right\rangle-\left\langle A u_{-}, v_{-}\right\rangle .
$$

The symbol $|\cdot|$ means the norm induced by this inner product.

From now on we are looking for a homeomorphism $h_{2}: V_{2} \subset \mathbb{H} \rightarrow h_{2}\left(V_{2}\right)$, with $V_{2} \subset V_{1}$, such that, if $\varphi=h_{1} \circ h_{2}$, then

$$
f(\varphi(x, y))=\frac{1}{2}\langle A x, x\rangle+f(g(y), y) .
$$

We will search $h_{2}$ of the form

$$
h_{2}(x, y)=x+\lambda(x, y)\left(x_{+}-x_{-}\right)+y,
$$

where $\lambda: \mathbb{H} \rightarrow[-1 / 2,1 / 2]$. Defining

$$
\psi(x, y)=\frac{1}{2}\langle A x, x\rangle+f(g(y), y), \quad \phi(x, y)=\psi(x, y)-f(x+g(y), y),
$$

we have

$$
\begin{aligned}
f(\varphi(x, y))= & f\left(x+\lambda(z)\left(x_{+}-x_{-}\right)+g(y), y\right) \\
= & \psi\left(x+\lambda(z)\left(x_{+}-x_{-}\right), y\right)-\phi\left(x+\lambda(z)\left(x_{+}-x_{-}\right), y\right) \\
= & \frac{1}{2}\left\langle A\left(x+\lambda(z)\left(x_{+}-x_{-}\right)\right), x+\lambda(z)\left(x_{+}-x_{-}\right)\right\rangle \\
& +f(g(y), y)-\phi\left(x+\lambda(z)\left(x_{+}-x_{-}\right), y\right) \\
= & \frac{1}{2}\langle A x, x\rangle+f(g(y), y)+\lambda(z)\left\langle A x, x_{+}-x_{-}\right\rangle \\
& +\frac{1}{2} \lambda(z)^{2}\left\langle A\left(x_{+}-x_{-}\right), x_{+}-x_{-}\right\rangle-\phi\left(x+\lambda\left(x_{+}-x_{-}\right), y\right) \\
= & \psi(x, y)+\lambda(z)|x|^{2}+\frac{1}{2} \lambda(z)^{2}\left\langle A\left(x_{+}-x_{-}\right), x_{+}-x_{-}\right\rangle \\
& -\phi\left(x+\lambda\left(x_{+}-x_{-}\right), y\right),
\end{aligned}
$$


116 A Morse lemma for degenerate critical points ...

and so, $f(\varphi(z))=\psi(z)$ if and only if $\lambda$ satisfies

$$
\phi\left(x+\lambda(z)\left(x_{+}-x_{-}\right), y\right)=\lambda(z)|x|^{2}+\frac{1}{2} \lambda(z)^{2}\left\langle A\left(x_{+}-x_{-}\right), x_{+}-x_{-}\right\rangle .
$$

Now observe that $\phi(0, y)=0$, thus any value we give for $\lambda(0, y)$ satisfies the last equation on these points, in particular $\lambda(0, y)=0$. For $x \neq 0$ and $y \in \mathbb{N}$ fixed, consider the function $\Gamma: \mathbb{R} \rightarrow \mathbb{R}$ defined by

$$
\Gamma(\lambda, x, y)=\frac{1}{2|x|^{2}}\left(2 \phi\left(x+\lambda\left(x_{+}-x_{-}\right), y\right)-\lambda^{2}\left\langle A\left(x_{+}-x_{-}\right), x_{+}-x_{-}\right)\right) .
$$

We want a neighborhood of 0 in $\mathbb{R} \times\left(\mathbb{N}^{\perp} \oplus \mathbb{N}\right)$ where $\Gamma$ is a contraction and, therefore, $\lambda=\lambda(x, y)$ will be a fixed point of $\Gamma$. We begin estimating $\Gamma^{\prime}$. Observe before that

$$
\frac{\partial \phi}{\partial x}(x, y) v=\langle A x, v\rangle-\frac{\partial f}{\partial x}(x+g(y), y) v
$$

hence $(\partial \phi / \partial x)(0, y)=0$ and, since $\phi^{\prime}$ is strongly differentiable at the origin, it follows that, given $\varepsilon>0$, if $\|x+y\|$ is small enough, $\|(\partial \phi / \partial x)(x, y)\| \leq \varepsilon|x|$. But

$$
\begin{aligned}
\left|\frac{\partial \Gamma}{\partial \lambda}(\lambda, x, y)\right| \leq & \frac{1}{|x|^{2}}\left\|\frac{\partial \phi}{\partial x}\left(x+\lambda\left(x_{+}-x_{-}\right), y\right)\right\|\left|x_{+}-x_{-}\right| \\
& +\frac{|\lambda|}{|x|^{2}}\left|\left\langle A\left(x_{+}-x_{-}\right), x_{+}-x_{-}\right\rangle\right| \\
\leq & k_{1} \varepsilon+k_{2}|\lambda|,
\end{aligned}
$$

where $k_{1}, k_{2}$ are constants. So, if we choose $\varepsilon$ small and $|\lambda| \leq 1 /\left(4 k_{2}\right)$, for instance, we will have $\left|\Gamma^{\prime}\right| \leq 1 / 2$ and, using the mean value theorem,

$$
\left|\Gamma(\lambda, x, y)-\Gamma\left(\lambda_{0}, x, y\right)\right| \leq \frac{1}{2}\left|\lambda-\lambda_{0}\right| \leq \frac{1}{2} .
$$

Observe that $\Gamma(0, x, y)=\phi(x, y) /|x|^{2}$. Then, taking $\lambda_{0}=0$, we get

$$
|\Gamma(\lambda, x, y)-\Gamma(0, x, y)| \leq \frac{1}{2}|\lambda| .
$$

Thus $|\Gamma(\lambda, x, y)| \leq\left.|\phi(x, y) /| x\right|^{2}|+(1 / 2)| \lambda \mid$. At last, if $|\lambda| \leq\left. 2|\phi(x, y) /| x\right|^{2} \mid$,

$$
|\Gamma(\lambda, x, y)| \leq 2\left|\frac{\phi(x, y)}{|x|^{2}}\right| .
$$

Therefore, if $\eta(x, y)=\left.2|\phi(x, y) /| x\right|^{2} \mid \leq 1 /\left(4 k_{2}\right), \Gamma$ is a contraction of the interval $[-\eta, \eta]$. But in fact $\lim _{z \rightarrow 0} \eta(x, y)=0$. Just observe that $\left(\partial^{2} \phi / \partial x^{2}\right)(0,0)=0$ as well as $\left(\partial^{2} \phi / \partial x \partial y\right)(0,0)=0$, thus, using the strong differentiability of $\partial \phi / \partial x$ at 0 , if $\|x+y\|$ is small enough

$$
\left\|\frac{\partial \phi}{\partial x}(x, y)\right\|=\left\|\frac{\partial \phi}{\partial x}(x, y)-\frac{\partial \phi}{\partial x}(0, y)\right\| \leq \varepsilon|x| .
$$


Now we just have to use the mean value inequality

$$
\|\phi(x, y)\|=\|\phi(x, y)-\phi(0, y)\| \leq|x| \sup \left\{\left\|\frac{\partial \phi}{\partial x}(t x, y)\right\| ; t \in[0,1]\right\} \leq \varepsilon|x|^{2} .
$$

The function $\lambda$ constructed in this way is bounded on an entire neighborhood of the origin and might be discontinuous only at the points $(0, y)$. Since $\lambda(x, y) \in$ $[-\eta(x, y), \eta(x, y)]$ we see it is continuous at 0 . However $h_{2}$ is continuous, even where $\lambda$ is not,

$$
\left|h_{2}(x, y)-h_{2}\left(0, y_{0}\right)\right|=\left|(1+\lambda) x_{+}\right|+\left|(1-\lambda) x_{-}\right|+\left|y-y_{0}\right| .
$$

As well as $h_{1}, h_{2}$ is differentiable at the origin and $d h_{2}(0)=I$. In fact, $\mid h_{2}(x, y)-$ $x-y|=| \lambda(x, y)|| x \mid$ and the differentiability follows from the continuity of $\lambda$ at 0 . Unfortunately, we cannot guarantee the strong differentiability of $h_{2}$ at 0 , what leads us to search the inverse $h_{3}$ of $h_{2}$ explicitly. Choosing a neighborhood of 0 in $\mathbb{H}$ in such a way that $|\lambda(x, y)|<1 / 2$, the function $h_{3}$ defined below is the function we are looking for

$$
h_{3}(x, y)=\frac{1}{1+\lambda(x, y)} x_{+}+\frac{1}{1-\lambda(x, y)} x_{-}+y .
$$

In fact, continuity is clear in the whole neighborhood except at the points $(0, y)$. For these we have

$$
\begin{aligned}
\left|h_{3}(x, y)-h_{3}\left(0, y_{0}\right)\right|^{2} & =\left|\frac{1}{1+\lambda(x, y)} x_{+}+\frac{1}{1-\lambda(x, y)} x_{-}+y-y_{0}\right|^{2} \\
& =\frac{\left|x_{+}\right|^{2}}{|1+\lambda(x, y)|^{2}}+\frac{\left|x_{-}\right|^{2}}{|1-\lambda(x, y)|^{2}}+\left|y-y_{0}\right|^{2},
\end{aligned}
$$

and so $h_{3}$ is continuous. That $h_{3}$ really is a local inverse of $h_{2}$ is just a simple verification of the equalities $h_{2} \circ h_{3}=i d=h_{3} \circ h_{2}$. Finally we see that $d h_{3}(0)=I$,

$$
\begin{aligned}
\left|h_{3}(x, y)-x-y\right|^{2} & =\left|\frac{x_{+}}{1+\lambda}-x_{+}\right|^{2}+\left|\frac{x_{-}}{1-\lambda}-x_{-}\right|^{2} \\
& =\frac{\lambda^{2}}{(1+\lambda)^{2}}\left|x_{+}\right|^{2}+\frac{\lambda^{2}}{(1-\lambda)^{2}}\left|x_{-}\right|^{2},
\end{aligned}
$$

and using the continuity of $\lambda$ at 0 again completes the proof.

It is not difficult to see that the Finsler energy verifies the hypothesis of this Morse lemma so a Morse theory can be developed as in the Riemannian case. Moreover, the index formula works the same as in the Riemannian case (see [5]) so we can repeat the proof of [3] obtaining the analogue of Theorem 1.1 for the Finsler case.

\section{Acknowledgement}

This work was partially supported by FAPESP and CAPES, Brazil. 
118 A Morse lemma for degenerate critical points ...

\section{References}

[1] R. Bott, Nondegenerate critical manifolds, Ann. of Math. (2) 60 (1954), 248-261. MR 16,276f. Zbl 058.09101.

[2] D. Gromoll and W. Meyer, On differentiable functions with isolated critical points, Topology 8 (1969), 361-369. MR 39\#7633. Zbl 212.28903.

[3] _ Periodic geodesics on compact Riemannian manifolds, J. Differential Geometry 3 (1969), 493-510. MR 41\#9143. Zbl 203.54401.

[4] F. Mercuri and G. Palmieri, Morse theory with low differentiability, Boll. Un. Mat. Ital. B (7) 1 (1987), no. 3, 621-631. MR 89f:58026. Zbl 633.58014.

[5] F. M. Souza, Geodésicas fechadas em variedades de Finsler, 1997, Doctoral Dissertation, UNICAMP.

Adriano Adrega de Moura: Instituto de Matemática, Estatística e Computaçăo CienTífica (IMECC), UniversidAde EstadUAL de CAMPINAS (UNICAMP), CP 6065, CEP 13083-970, CAMPINAS, SP, BRAZIL

E-mail address: adrianoam@ime.unicamp.br

Fausto Marçal de Souza: Instituto de Matemática e Estatística (IME), Universidade Federal de Goiás (UFG), CP 131, CEP 74001-970, GO, BRAZIL

E-mail address: fausto@mat.ufg.br 


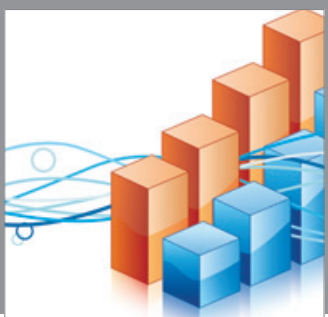

Advances in

Operations Research

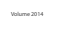

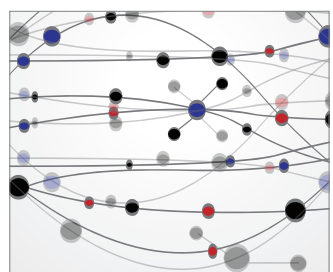

\section{The Scientific} World Journal
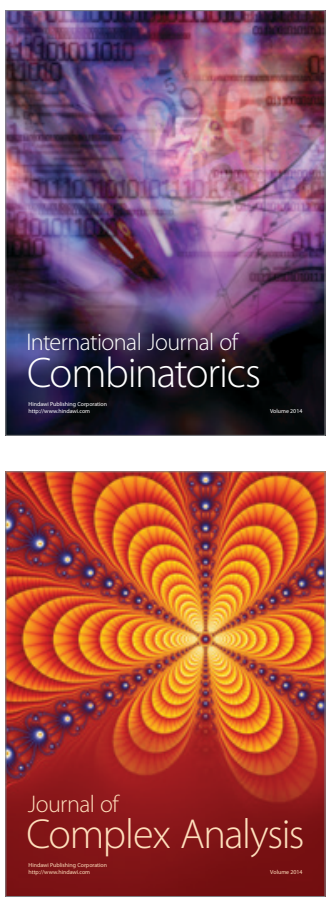

International Journal of

Mathematics and

Mathematical

Sciences
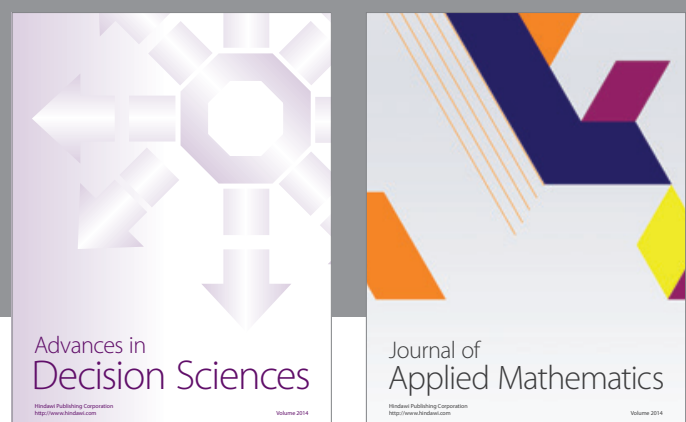

Journal of

Applied Mathematics
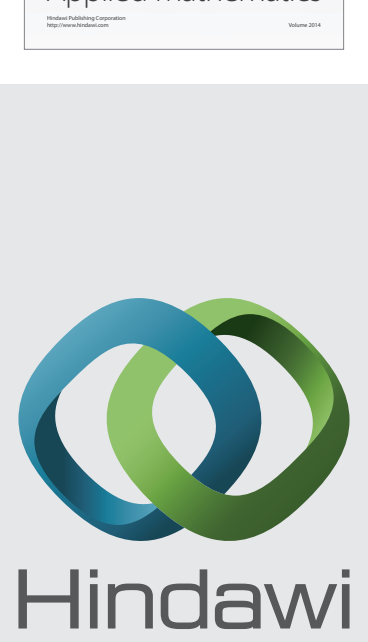

Submit your manuscripts at http://www.hindawi.com
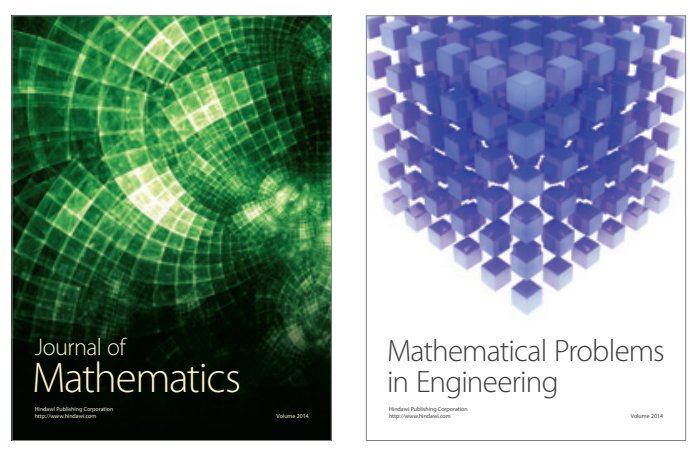

Mathematical Problems in Engineering
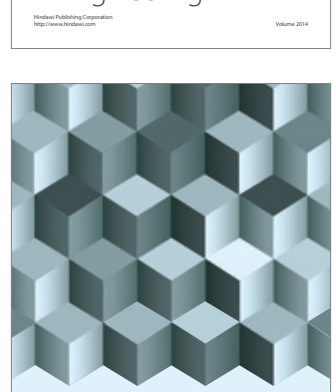

Journal of

Function Spaces
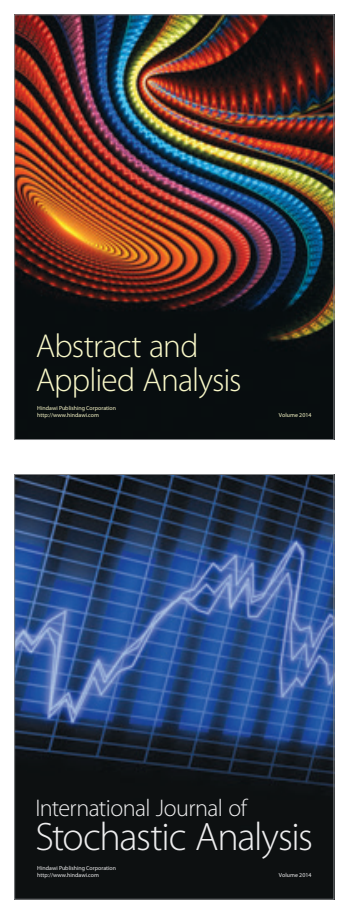

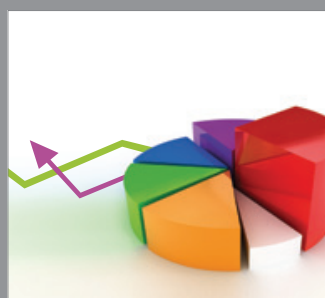

ournal of

Probability and Statistics

Promensencen
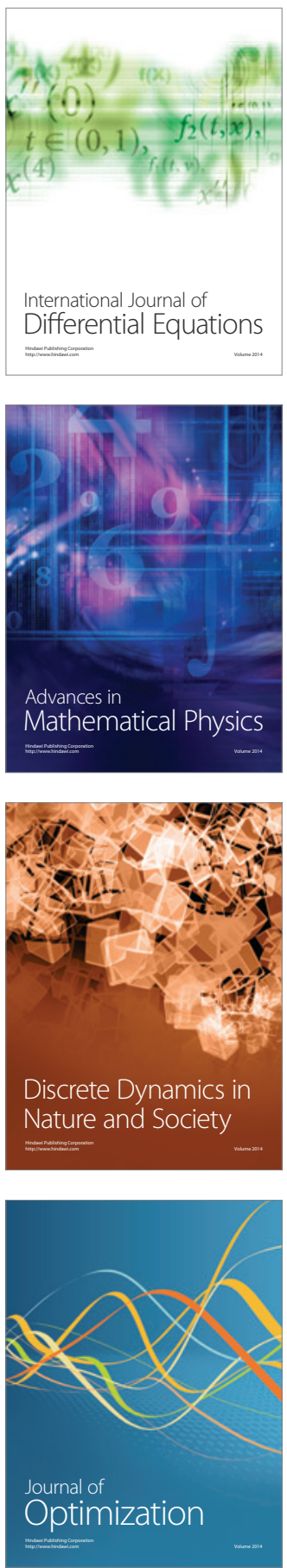